nutzen, der zusätzliche investive Kosten nicht rechtfertigt", so Herth. Glücklicherweise gehöre das NBI heute standardmäßig zur Ausrüstung in modernen Bronchoskopen, sodass es bei Bedarf zugeschaltet werden kann, ohne dass Kosten entstehen [4].

\section{Konfokale Laserendomikroskopie}

Eine Technologie, die immer weiter in den Fokus rückt, ist die konfokale Laserendomikroskopie. Das Verfahren eignet sich für die Emphysem-Diagnostik, genauer gesagt für die Vermessung der peripheren Alveolen. In einer Studie an 15 Emphysem-Patienten und 15 Gesunden fand sich eine hohe Korrelation zwischen dem Atemwegsdurchmesser auf alveolärer Ebene und dem Tiffeneau-Index sowie dem CT-Befund [5]. „Man kann das Emphysem jetzt auch endoskopisch sehen, aber es bleibt die Frage, welchen Zusatznutzen diese Technik liefert“, so Herth. Auch bei der Beurteilung der Dignität von Rundherden konnte damit bisher kein Vorteil gegenüber dem bisherigen Standard belegt werden [6].

Dr. med. Peter Stiefelhagen
Literatur:

1. Girard P et al. Clin Lung Cancer 2017;18(5):512-18

2. Macedo P et al. Respir Med 2017: 127:40-4

3. Jiao AX et al. World J Pediatr 2017: doi: 10.1007/s12519-0170046-1

4. Tremblay A et al. Chest 2016: 150(5):1015-22

5. Yserbyt J et al. Thorax 2017: doi: 10.1136/thoraxjnl-2016-209746

6. Seth S et al. Sci Rep 2016:6:31372

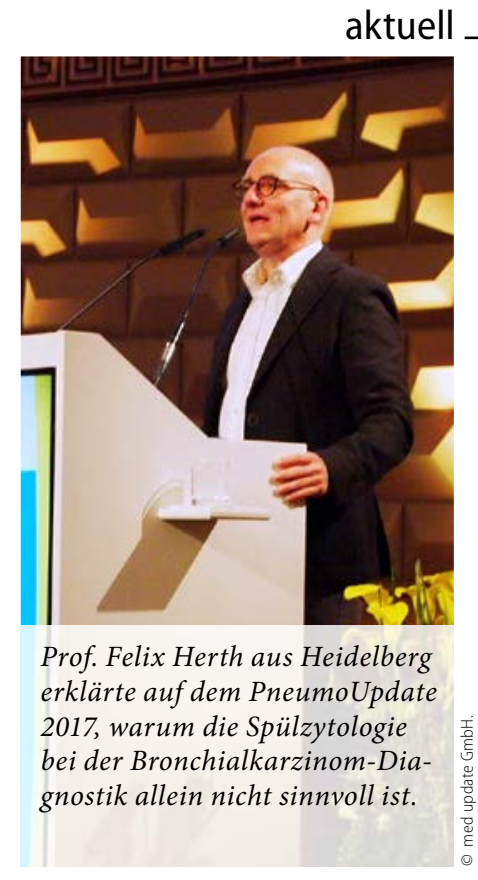

\title{
Therapie bei Pleuramesotheliom wird immer besser
}

\section{Das Pleuramesotheliom hat eine schlechte Prognose. Erste Ergebnisse mit Immuntherapien lassen aber zu- mindest für einige Patienten auf Vorteile hoffen.}

Das Pleuramesotheliom wird meist in einem Stadium diagnostiziert, wenn eine kurative Behandlung nicht mehr möglich ist. Für die meisten Patienten bleibt somit nur die palliative Therapie. Die Standardtherapie bei Ergussbildung ist die Pleurektomie bzw. Dekortikation mit anschließender palliativer Chemotherapie mit Platin und Pemetrexed. Der Stellenwert der Radiotherapie ist umstritten, zumal der Tumor kaum strahlensensibel ist. Ein ausgedehnter operativer Eingriff in Form einer Pleuropneumonektomie unter Mitnahme des Perikards kommt nur in Einzelfällen in einem Frühstadium bei Patienten in einem sehr guten Allgemeinzustand in Betracht.

\section{Checkpoint-Inhibitoren und VEGF-Antikörper}

$\mathrm{Da}$ in Folge des Asbestverbots die Zahl jüngerer Patienten abnimmt, wird das Patientenkollektiv immer älter. „Die älteren Patienten sind oft nicht mehr fit genug für eine cisplatinhaltige multimodale Therapie“, so Prof. Martin Reck aus Großhansdorf. Daher brauche man dringend besser verträgliche Therapieoptionen. Dazu gehören antiangiogenetisch wirksame Substanzen und Immunonkologika, also Checkpoint-Inhibitoren.

In der Phase-III-Studie MAPS wurden 448 Patienten entweder nur mit Cisplatin und Pemetrexed behandelt oder sie erhielten zusätzlich den VEGF-Antikörper Bevacizumab. Der primäre Endpunkt wurde durch Bevacizumab von 16,1 auf 18,8 Monate verlängert (HR: 0,77; $p=0,0167)$. Doch die Rate an schweren Komplikationen war im Bevacizumab-Arm höher als unter der alleinigen Chemotherapie (71 vs. $62 \%$ ) [1].

In einer Phase-II-Studie wurde dieselbe Chemotherapie mit dem Multikinase-Inhibitor Nintedanib kombiniert. Mit dieser innovativen Substanz verlängerte sich das progressionsfreie Überleben (PFS) von 5,4 auf 9,7 Monate (HR: 0,54, p=0,013). Auch beim Gesamtüberleben fand sich ein Trend zugunsten von Nintedanib (14,2 vs. 18,3 Monate; HR: 0,77, p =0,3193). Die einzige schwerwiegende Nebenwirkung, die mit der Nintedanibtherapie signifikant häufiger auftrat, war die Neutropenie (43,2 vs. $12,2 \%)$ [2].

Auch die Daten zur Immuntherapie sind vielversprechend. „Zwar profitieren längst nicht alle Patienten davon, so Reck. Aber wenn sie ansprechen, sei es oft ausgeprägt und längerfristig. Welche Rolle dabei die PD-L1-Expression spiele, könne man bisher nicht sagen. In einer randomisierten Phase-II-Studie mit 114 Patienten wurde der PD-1-Inhibitor Nivolumab oder die Kombination von Nivolumab plus Ipilimumab in der Zweitoder Drittlinientherapie untersucht. Durch den Einsatz der Checkpoint-Inhibitoren wurde eine Krankheitskontrollrate von 39,7\% unter der Nivolumab-Monotherapie und von 51,6\% mit der Kombination erreicht. Das Gesamtüberleben lag mit der Monotherapie im Median bei 13,6 Monaten und war bei der Kombination noch nicht erreicht. Die häufigsten Nebenwirkungen waren Asthenie, Fatigue und Durchfälle [3].

In einer Phase-Ib-Studie wurde Pembrolizumab als Monotherapie bei 25 Patienten mit einer PD-L1-Expression eingesetzt. Die Ansprechrate lag bei $20 \%$. 52\% der Patienten zeigten eine Krankheitsstabilisierung. Die Ansprechdauer lag im Mittel bei 12 Monaten. Bei $64 \%$ der Patienten traten Nebenwirkungen auf wie Fatigue, Übelkeit und Gelenkschmerzen. Schwerwiegende Nebenwirkungen waren aber selten [4]. Dr. med. Peter Stiefelhagen

Literatur:

1. Zalcman G et al. Lancet 2016; 387:1405-14

2. Grosso F et al. J Clin Oncol 2017; doi: 10.1200/JCO.2017.72.9012

3. Zalcman G et al. Ann Oncol 017; 28:605-49

4. Alley EW et al. Lancet Oncol 2017; 18(5):623-30 